Contrib. Plasma Phys. vol (year) num, $\mathrm{p}-\mathrm{p}$

\title{
Practical Improvements to the Lee-More Conductiv- ity Near the Metal-Insulator Transition
}

\author{
M. P. DESJaRlais \\ Sandia National Laboratories, Albuquerque, New Mexico, USA 87185-1186 \\ e-mail: mpdesja@sandia.gov
}

\begin{abstract}
The wide-range conductivity model of Lee and More [1] is modified to allow better agreement with recent experimental data and theories for dense plasmas in the metal-insulator transition regime. Modifications primarily include a new ionization equilibrium model, consisting of a smooth blend between single ionization Saha (with a pressure ionization correction) and the generic Thomas-Fermi ionization equilibrium, a more accurate treatment of electron-neutral collisions using a polarization potential, and an empirical modification to the minimum allowed collision time. These simple modifications to the Lee-More algorithm permit a more accurate modeling of the physics near the metal-insulator transition, while preserving the generic Lee-More results elsewhere.
\end{abstract}

\section{Introduction}

High fidelity computational modeling of plasma formation and evolution, such as that resulting from high current densities in metal, requires accurate and wide-range electrical and thermal conductivity models. Of particular importance for simulating many modern high-energy-density physics experiments is the metal-insulator transition regime with temperatures on the order of one-half to several $\mathrm{eV}$ and densities at, or below, solid. The two most commonly used wide-range conductivity models for these computations are the Lee-More [1] algorithm for in-line calculations and the SESAME (Rinker) [2] tabular conductivities. These models were primarily developed for higher density and temperature regimes and are, not unexpectedly, grossly inaccurate for relatively low temperatures (below $3 \mathrm{eV}$ ) and solid to sub-solid densities. Recent experiments $[3,4,5]$ and theoretical analyses $[6,7]$ of the electrical conductivity of dense metal plasmas in the vicinity of the metal-insulator transition are in mutual agreement, but clearly illustrate differences of several orders of magnitude with the Lee-More and SESAME conductivities. We have recently developed a modified Lee-More algorithm that smoothly joins the generic Lee-More results outside of this regime, but is modified in such a way as to permit good agreement with the experimental and theoretical results in the transition regime. This extension of the Lee-More model also gives good agreement with recent aluminum data obtained at somewhat higher temperatures [8].

\section{Modifications to the Lee-More Algorithm}

Using the notation of Lee and More [1], the electrical conductivity may be written $\sigma=A\left(\mu / k_{b} T, \omega \tau\right) n_{e} e^{2} \tau / m_{e}$ where $n_{e}$ is the electron number density, $e$ is the electron charge, $m_{e}$ is the electron mass, and $\tau$ is the electron relaxation time. The multiplier $A$ is a two-dimensional function of the electron chemical potential and magnetization 


\section{DISCLAIMER}

This report was prepared as an account of work sponsored by an agency of the United States Government. Neither the United States Government nor any agency thereof, nor any of their employees, make any warranty, express or implied, or assumes any legal liability or responsibility for the accuracy, completeness, or usefulness of any information, apparatus, product, or process disclosed, or represents that its use would not infringe privately owned rights. Reference herein to any specific commercial product, process, or service by trade name, trademark, manufacturer, or otherwise does not necessarily constitute or imply its endorsement, recommendation, or favoring by the United States Government or any agency thereof. The views and opinions of authors expressed herein do not necessarily state or reflect those of the United States Government or any agency thereof. 


\section{DISCLAIMER}

Portions of this document may be illegible in electronic image products. Images are produced from the best available original document. 
and has a minimum value of 1.0 in the fully magnetized $(\omega \tau \rightarrow \infty)$ and/or fully degenerate $\left(\mu / k_{b} T \rightarrow \infty\right)$ limits, and a maximum value of $32 / 3 \pi$ in the non-degenerate, unmagnetized limit $\left(\mu / k_{b} T \rightarrow-\infty, \omega \tau \rightarrow 0\right)$.

With the generic Lee-More model, the electron density is usually determined using a Thomas-Fermi ionization model. This is especially appealing for its natural connection to Thomas-Fermi based equations-of-state and its complete coverage of density and temperature. However, the Thomas-Fermi model is inadequate for capturing the metal-insulator transition since it neglects any atomic structure effects on the ionization equilibrium and thus gives much too high an ionization level under conditions that are well known to produce very low, insulator-like, ionization levels. The model used here simply makes a weighted blend of Thomas-Fermi and a single ionization Saha model with a pressure ionization correction. The resulting model is completely global, and smoothly transitions from Thomas-Fermi to non-ideal Saha in those regions where the ionization calculated from the non-ideal Saha model is significantly less than 1.0. The Saha contribution is determined from

$$
f_{e}=\frac{1}{2}\left(\sqrt{K^{2}+4 K}-K\right)
$$

where

$$
K=\frac{2 g_{1}}{g_{0}} \frac{1}{n_{a}}\left(\frac{2 \pi m_{e} k_{b} T}{h^{2}}\right)^{\frac{3}{2}} \exp \left[-\frac{I}{k_{b} T}\left(1-\left(\frac{1.5 e^{2}}{I R_{a}}\right)^{\frac{3}{2}}\right)\right]
$$

The statistical weights $g_{0}$ and $g_{1}$ correspond to the ground state of the neutral atom and singly ionized ion respectively, $I$ is the first ionization energy, $R_{a}=\left(3 / 4 \pi n_{a}\right)^{1 / 3}$ is the Wigner-Seitz radius, $n_{a}$ is the total neutral plus ion number density. For both $\mathrm{Cu}$ and $\mathrm{Al}, g_{0}=2$ and $g_{1}=1$, and in general the level degeneracy $g=2 J+1$ is used. The second term in parentheses in the exponential gives a semi-empirical pressure ionization correction. The factor 1.5 and the exponent $3 / 2$ were suggested through comparisons with Redmer's [6] PIP model calculations. Note that the argument of the exponential changes sign at $1.5 e^{2} / I R_{a}=1$. This is consistent with Likalter's [9] percolation criterion $\left(e^{2} / I R_{a}\right)^{3} \approx 1 / 3$ for the metal-insulator transition. A smooth blend with the Thomas-Fermi ionization level $Z_{\mathrm{TF}}[10]$ is obtained with

$$
\bar{Z}=f_{e}^{2 / Z_{\mathrm{TF}}^{2}} Z_{\mathrm{TF}}+\left(1-f_{e}^{2 / Z_{\mathrm{TF}}^{2}}\right) f_{e} ; \quad n_{e}=\bar{Z} n_{a} .
$$

The generic Lee-More algorithm includes the electron-neutral scattering with an ad hoc collision cross section, typically with a default value of $2 \times 10^{-15} \mathrm{~cm}^{2}$. This fixed cross section is replaced here with a momentum transfer cross section calculated in the Born approximation using a screened polarization potential $[6,7]$. The resulting values are both density and temperature dependent, and may be substantially larger than $2 \times 10^{-15} \mathrm{~cm}^{2}$, particularly in those regions where the ionization is low and the electron-neutral collisions have a significant impact on the conductivity. Typical values for the average cross section for $\mathrm{Al}$ or $\mathrm{Cu}$ at $10000 \mathrm{~K}$, and densities about two orders of magnitude below solid, are about $30 \times 10^{-15} \mathrm{~cm}^{2}$. Still greater are the cross sections for the alkali metals (other than hydrogen) because of their large dipole polarizabilities. In order to streamline the use of these cross sections for in-line calculations, we have derived an accurate fit to the cross section. Following the definitions and notation in Refs. $[6,7]$, the electron-neutral momentum transfer cross section is approximately

$$
\Sigma_{e 0}=\frac{\pi^{3}\left(\alpha_{D} / 2 r_{0} a_{B}\right)^{2}}{A_{\kappa}^{2}+3 B_{\kappa} k r_{0}+7.5 C_{\kappa}\left(k r_{0}\right)^{2}-3.4 D_{\kappa}\left(k r_{0}\right)^{3}+10.6668 E_{\kappa}\left(k r_{0}\right)^{4}}
$$


where $\kappa$ is the inverse screening length, $k$ is the electron wave number, $\alpha_{D}$ is the dipole polarizability, $a_{B}$ is the Bohr radius, $r_{0}=\sqrt[4]{\alpha_{D} a_{B} / 2 Z^{1 / 3}}$ is the cutoff radius, and

$$
\begin{gathered}
A_{\kappa}=1+2 \kappa r_{0}+\frac{7}{\pi^{2}}\left(\kappa r_{0}\right)^{2}+\frac{\pi}{7}\left(\kappa r_{0}\right)^{3}, \quad B_{\kappa}=\exp \left(-18 \kappa r_{0}\right) \\
C_{\kappa}=\frac{1+22 \kappa r_{0}-11.3\left(\kappa r_{0}\right)^{2}+33\left(\kappa r_{0}\right)^{4}}{1+6 \kappa r_{0}+4.7\left(\kappa r_{0}\right)^{2}+2\left(\kappa r_{0}\right)^{4}}, \\
D_{\kappa}=\frac{1+28 \kappa r_{0}+13.8\left(\kappa r_{0}\right)^{2}+3.2\left(\kappa r_{0}\right)^{3}}{1+8 \kappa r_{0}+10\left(\kappa r_{0}\right)^{2}+\left(\kappa r_{0}\right)^{3}}, \quad E_{\kappa}=1+0.1 \kappa r_{0}+0.3665\left(\kappa r_{0}\right)^{2} .
\end{gathered}
$$

A sufficiently accurate distribution-averaged cross section for in-line calculations $\bar{\Sigma}_{e 0}$ is obtained with $k=m_{e} \bar{V}_{e} / \hbar$, where $\bar{V}_{e}$ is the average electron velocity.

The Lee-More algorithm includes a lower bound to the electron relaxation time determined from $\tau_{\min }=p_{2} R_{a} / \bar{V}_{e}$, where $p_{2}$ is a fit parameter of order unity. This lower bound on the electron relaxation time tends to dominate the conductivity in the region of the metal insulator transition, and in this form precludes a reasonable approximation to the data. DeSilva and Katsouros [5] have noted that examination of their data for several metals reveals an approximately universal scaling of conductivity with $n_{a}$, independent of temperature, for temperatures below about an $\mathrm{eV}$. This suggests an empirically motivated replacement for $p_{2}$ of the form

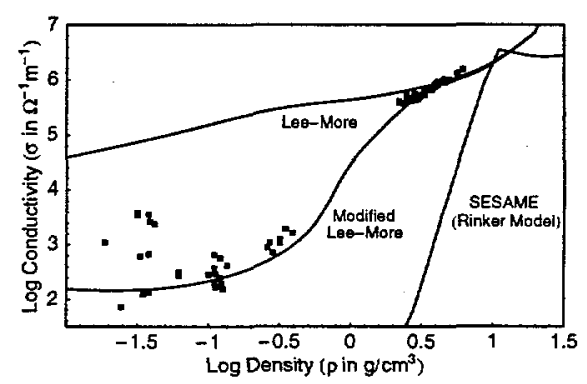

Fig. 1: A comparison between the experimental results of De Silva et al. [3], the Lee-More and SESAME (Rinker) conductivities, and the result of our modifications to Lee-More, all for copper at $6000 \mathrm{~K}$.

$$
p_{2}=\min \left[p_{2 a}+\frac{p_{2 b}}{\Theta},\left(\frac{n_{a}}{2 \cdot 10^{22} \mathrm{~cm}^{-3}}\right)^{2 / \Theta}\right]
$$

where $\Theta=1+\exp [(T-25000 \mathrm{~K}) / 10000 \mathrm{~K}]$ is a blending function and $p_{2 a}$ and $p_{2 b}$ are constants of order unity that may be chosen to fine tune the model to measured conductivities. For $\mathrm{Cu},\left(p_{2 a}, p_{2 b}\right)=(0.65,1.8)$, and for $\mathrm{Al},\left(p_{2 a}, p_{2 b}\right)=(0.65,3.0)$, are reasonable choices.

In addition to the above changes to the Lee-More algorithm, electron-electron scattering has been added following the treatment in Ref. [11] and the inverse Fermi functions needed for calculation of the chemical potential $\mu$ and the inverse screening length $\kappa$ are evaluated accurately using Zimmerman's approximations [12].

\section{Comparisons with data}

An example of the inaccuracies that can result when using either the Lee-More or SESAME-Rinker conductivities in the insulator regime is illustrated in Fig. 1 . The plot shows a comparison between these popular wide-range models and recent data for copper at $6000 \mathrm{~K}$ [3]. Note the logarithmic scale for the conductivity. The conductivities in the insulator regime are severely underestimated by the SESAME tabular 
values, and overestimated by two orders of magnitude with the Lee-More algorithm. Also shown in Fig. 1 is the curve for $\mathrm{Cu}$ at $6000 \mathrm{~K}$ obtained with our modified Lee-More algorithm. The modifications generate good agreement with the measured conductivities over the entire range shown.

In Fig. 2, three isotemperature contours are shown for comparison with aluminum conductivity data at 10,20 , and $30 \mathrm{kK}$ [3]. Also shown is data for temperatures ranging from 0.86 to $24.6 \mathrm{eV}$ [8] plotted parametrically with temperature along with the corresponding values calculated with this model. The agreement in all cases is quite good.

Numerical simulations of conductors subject to ultra-high current densities, using new SESAME format files generated with this model, show dramatically different behavior compared to the older SESAME files [13].

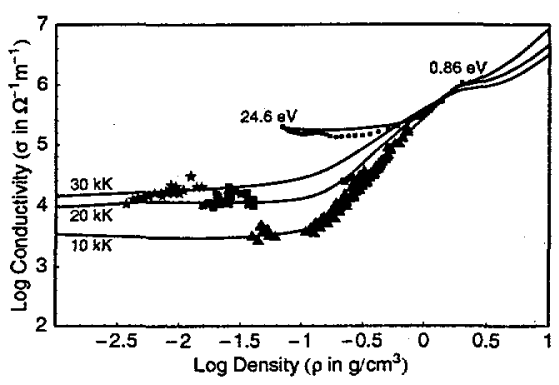

Fig. 2: A comparison between the results of the modified Lee-More algorithm for aluminum and the data of De Silva, et al. [3], and Benage, et al. [8]

\section{Acknowledgements}

I would like to thank Ronald Redmer, Sandra Kuhlbrodt, Alan DeSilva, and John Benage for several useful conversations, and for the results of their theoretical and experimental studies. Sandia is a multiprogram laboratory operated by Sandia Corporation, a Lockheed Martin Company, for the United States Department of Energy under Contract No. DE-AC04-94AL85000.

\section{References}

[1] LEE, Y.T., MORE, R.M., Phys. Fluids 27(1984)1273

[2] Rinker, G.A., Phys. Rev. A 37(1988)1284

[3] DeSilva, A.W., Katsouros, J.D., Phys. Rev. E 57(1998)5945

[4] KirSCh, I., Kunze, H.-J., Phys. Rev. E 57(1998)6557

[5] DeSilva, A.W., Katsouros, J.D., J. Phys. IV France 10(2000)Pr5-65

[6] REDMER, R., Phys. Rev. E 59(1999)1073

[7] Kuhlbrodt, S., Redmer, R., Phys. Rev. E 62(2000)

[8] Benage, J.F., Shanahan, W.R., Murillo, M.S., Phys. Rev. Lett. 83(1999)2953

[9] Likalter, A., Phys. Scr. 55(1997) 114

[10] More, R.M., Adv. At. Mol. Phys. 21(1985)305

[11] Ebeling, W., Förster, A., Fortov, V.E., Gryaznov, V.K. Polishchuk, A.YA., Thermophysical Properties of Hot Dense Plasmas, Teubner Verlagsgesellschaft, Stuttgart (1991) pp. 194-222.

[12] Zimmerman, R., Many-Particle Theory of Highly Excited Semiconductors, Teubner Verlagsgesellschaft, Leipzig (1988) p. 150.

[13] Rosenthal, S.E., Desjarlais, M.P., Spielman, R.B., et al, to be published in IEEE Trans. Plasma Sci. (2000)

Received August 30, 2000. 